\title{
О числе топологически неэквивалентных функций с одной вырожденной критической точкой типа седло на двумерной сфере, II
}

\section{Александр Анатольевич Кадубовский}

Аннотация В работе рассматривается класс гладких функций с тремя критическими значениями на двумерной сфере $S^{2}$, у которых кроме $M$ локальных максимумов и $m$ локальных минимумов только одна критическая точка типа седло. Для функций из указанного класса представлен полный топологический инвариант, с помощью которого для произвольных натуральных $M$ и $m$ установлены явные формулы для подсчета числа топологически неэквивалентных (в двух различных аспектах) таких функций.

Ключевые слова smooth function, saddle critical point, surface, topological classification, 2-color chord diagram, non-crossing partition, Narayana number.

УДК 517.938.5 + 519.514.17

Посвящается памяти моего научного руководителя Владимира Васильевича Шарко

\section{Введение}

Представленная статья является логическим завершением цикла авторских работ [14]-[17], посвященных получению явных формул для подсчета точного числа топологически неэквивалентных (в двух различных аспектах) гладких функций определенного класса на двумерной сфере $S^{2}$. 
Пусть $(N, \partial N)$ - гладкая поверхность с краем $\partial N$ ( $\partial N$ может быть ). Обозначим через $C^{\infty}(N)$ пространство бесконечно дифференцируемых функций на $N$ с краем $\partial N=\partial_{-} N \bigcup \partial_{+} N$, все критические точки которых изолированы и лежат во внутренности $N$ на одной линии уровня, а на компонентах связности края $\partial_{-} N\left(\partial_{+} N\right)$ они принимают одинаковое значение $a(b)$.

Функции $f$ и $g$ из $C^{\infty}(N)$ называют топологически эквивалентными, если существуют гомеоморфизмы $h: N \rightarrow N$ и $l: R^{1} \rightarrow R^{1}$ ( $l$ сохраняет ориентацию), такие что $g=l \circ f \circ h^{-1}$. Если $h$ сохраняет ориентацию, функции $f$ и $g$ будем называть $O$-топологически эквивалентными. В дальнейшем также будем полагать, что $N$ - замкнутая ориентируемая поверхность.

Пусть $f \in C^{\infty}(N)$, а $x_{0} \in N$ - ее изолированная критическая точка, не являющаяся локальным экстремумом. Если топологический тип линий уровня при переходе через $x_{0}$ меняется (не меняется), то такую точку $x_{0}$ (следуя [18], [11]) называют существенно (соответственно несущественной) критической точкой.

Хорошо известно [5], что в некоторой окрестности своей существенно критической точки $x_{0}$ функция $f$ топологически эквивалентна функции $f_{n}(x ; y)=\operatorname{Re}(x+i y)^{n}+c_{n}$, причем $n \geq 2$ (непрерывной заменой координат приводится к виду $\left.f_{n}=\operatorname{Re} z^{n}+c_{n}\right)$. Более того, локально, функции классифицируются целым числом - индексом Пуанкаре. Напомним, что индексом Пуанкаре $\operatorname{ind}^{f}\left(x_{0}\right)$ критической точки $x_{0}$ функции $f$ называют индекс Пуанкаре ее поля градиента $\operatorname{grad} f$ для некоторой римановой метрики. Также известно, что для функции $f=\operatorname{Re} z^{n}$ индекс Пуанкаре равен $\operatorname{ind}^{f}(0)=1-n$, а для каждой критической точки на поверхности $\operatorname{ind}^{f}\left(x_{0}\right) \leq 1$ [5].

Число $k$ существенно критических точек $x_{j}$ функции $f$ вместе со значениями $n_{j}$ (показателями в представлениях $f$ в виде $f_{j}=\operatorname{Re} z^{n_{j}}+c_{n_{j}}$ в окрестностях $x_{j}$ ) называют топологическим сингулярным типом функции $f$.

В работе В.В. Шарко [18] изучен вопрос топологической эквивалентности функций из класса $C^{\infty}(N)$. Ним установлено, что существует лишь конечное число топологически неэквивалентньх функций с фиксированным сингулярным типом, равное числу неизоморфньх цветных спин-графов, расширения которых гомеоморфны поверхности $N$. Однако неизвестно сколько таких классов эквивалентности. В общем случае эта задача оказалась очень сложной и остается нерешенной к этому времени проблемой. 


\section{1 Постановка задачи}

Если же ограничиться рассмотрением класса функций $C_{M, m}\left(N_{g}\right)$ с тремя критическими значениями на замкнутой ориентируемой поверхности $N_{g}$ (рода $g \geq 0$ ), у которых кроме $M$ локальных максимумов и $m$ локальных минимумов только одна сушественно критическая точка $x_{0}$ (в дальнейшем - вырожденная критическая точка типа седло), то упомянутая выше задача несколько упрощается.

А именно, как следует из работы [4], для функций из класса $C_{M, m}\left(N_{g}\right)$ топологическим инвариантом является индекс Пуанкаре единственной критической точки $x_{0}$ типа седло, который равен

$$
\text { ind }^{f}\left(x_{0}\right)=2-2 g-M-m \text {. }
$$

Полным топологическим инвариантом для функций из класса $C_{M, m}\left(N_{g}\right)$ является, так называемая, 2-цветная $O$-диаграмма с

$$
n=2 g-1+M+m
$$

хордами, которая содержит $M$ черных (бельх) и $m$ белых (черных) циклов. Кроме того, в работах автора [11], [12] полностью решена задача о подсчете числа $O$-топологически неэквивалентных функций из класса $C_{1,1}\left(N_{g}\right), g \geq 1$.

Однако, в общем случае (для произвольных натуральных $M, m$ и $g \geq 0)$, подсчет числа топологически неэквивалентных функций из класса $C_{M, m}\left(N_{g}\right)$ также оказался достаточно сложной комбинаторной задачей. Даже для функций на двумерной сфере $S^{2}$ вопрос оставался открытым.

Формулы (в явном виде) для подсчета числа $O$-топологически неэквивалентных функций из класса $C_{M, m}\left(S^{2}\right)$ раннее были известны только для начальных $M$ и произвольных $n \geq M$ (или, что тоже, для начальных $M$ и натуральных $m=n+1-M)$, а именно:

$$
\begin{aligned}
& \text { для } M=1,2,3,4 \text { и } \forall n \geq M \text { формулы были получены в работе [14]; } \\
& \text { для } M=5,6 \text { и } \forall n \geq M-\text { в работе [15]; } \\
& \text { для } M=7,8 \text { и } \forall n \geq M-\text { в работе [16]; } \\
& \text { для } M=9 \text { и } \forall n \geq M-\text { в работе [17]. }
\end{aligned}
$$

Модернизации полного топологического инварианта для функций из класса $C_{M, m}\left(S^{2}\right)$, возможным его интерпретациям, а также получению формул для подсчета точного числа $O$-топологически (топологически) неэквивалентных таких функций для произвольных $M, m \in \mathrm{N}$ и посвящена данная статья. 


\section{2-цветные хордовые диаграммы: основные понятия и определения}

Напомним (напр. [3]), что хордовой диаграммой порядка $n$ или, коротко, $n$ диаграммой называют конфигурацию на плоскости, состоящую из окружности, $2 n$ точек на ней (являющихся вершинами правильного $2 n$-угольника) и $n$ хорд, соединяющих указанные точки. Хордовые диаграммы называют изоморфными, если одну можно получить из другой в результате поворота. Диаграммы называют эквивалентными, если их можно совместить в результате поворота, зеркальной симметрии, или же их композиции.

Определение 1 Окружность с 2 почками на ней (являющихся вершинами правильного 2 -уголъника), дуги которой поочередно раскрашены в два ивета (черный и белый) и фиксированной нумерацией указанных вершин (числами от 1 до 2 п по часовой стрелке) будем называть 2-цветным $2 n$-иаблоном - рис. 1 a).

2-цветной n-диаграммой будем называть хордовую n-диаграмму, построенную на основе 2-цветного 2 -шаблона.

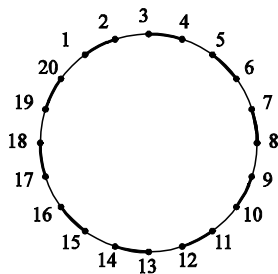

a)

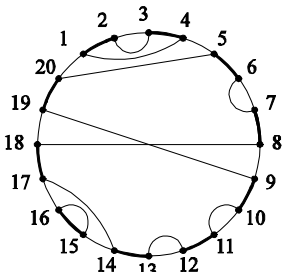

b)

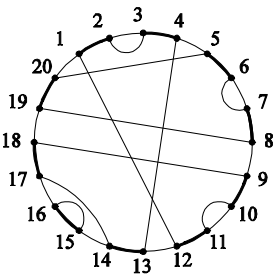

c)

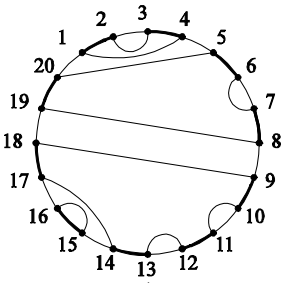

d)

Рис. 1

a) 2-цветный 20 -шаблон;

b) $N$-диаграмма (с 10 хордами), у которой 7 белых и 3 черных циклов;

c) $O$-диаграмма (с 10 хордами), у которой 6 белых и 3 черных циклов;

d) планарная $O$-диаграмма (с 10 хордами), у которой 7 белых и 4 черных циклов

Определение 2 2-цветную $n$-диаграмму, которая не содержит (содержит) хорд, соединяющих вершины с номерами одинаковой четности, будем называть O-диаграммой (N-диаграммой) - рис. 1 b), с). Множество 2-цветных О-диаграмм с $n$ хордами обозначим через $\Im_{n}^{O}$.

Определение 3 b-циклом (w-циклом) диаграммы из класса $\Im_{n}^{O}$ будем называть чередующуюся последовательность хорд и черных (соответственно бельх) дуг, которые образуют гоммеоморфный образ (ориентированной) окружности - рис. 1 b) $-d$ ). 
Пусть $\lambda$ - число черных и белых циклов диаграммы из $\Im_{n}^{O}$. Тогда родом диаграммы следует считать (напр. [3], [13]) целое число $g$, определяемое соотношением

$$
g=\frac{n-\lambda+1}{2} .
$$

Определение 4 Диаграмму из класса $\Im_{n}^{O}$, род которой равен нулю, будем называть диаграммой минимального рода или, коротко, планарной диаграммой - pис. 1 d). Подмножество планарных диаграмм из класса $\Im_{n}^{O}$, которье содержат точно $k(1 \leq k \leq n)$ черных (или, что тоже, $n-k+1$ белых) ииклов обозначим $\Im_{k, n}$.

Хорошо известно (напр. из [3], [2]), что $n$-диаграмма (по сути из $\Im_{n}^{O}$ ) является планарной тогда и только тогда, когда она не содержит пересекающихся хорд.

Определение 5 Как и в случае (нераскрашенных) хордовых $n$-диаграмм, 2-цветные диаграммы будем называть

изоморфными, если их можно совместить в результате поворота (по часовой стрелке) около общего центра;

эквивалентными, если их можно совместить в результате зеркальной симметрии и/или поворота около общего центра.

Известно (напр. из [3], [11]), что $n$-диаграмму (в том числе из $\Im_{n}^{O}$ ) можно отождествить с ее «склейкой» - подстановкой-инволюцией $\alpha$, столбцы которой - суть номера вершин, инцидентных одной хорде. Так, например, диаграмме на рис. $1 d$ ) соответствует подстановка

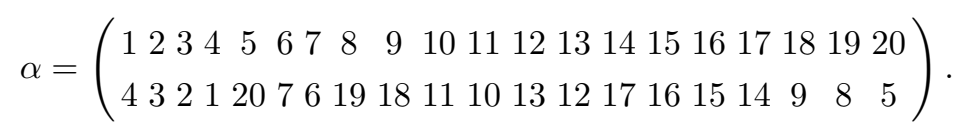

Более того, как следует из работ [3], [11], циклическая группа $C_{2 n}^{*}$ порядка $n$, порожденная перестановкой

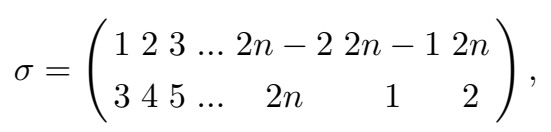

действует на множестве склеек диаграмм из $\Im_{n}^{O}$ как сопряжение, а именно:

диаграммы $D_{1}\left(\alpha_{1}\right)$ и $D_{2}\left(\alpha_{2}\right)$ изоморфны тогда и только тогда, когда существует $\gamma \in C_{2 n}^{*}$ такая, что $\alpha_{1}=\gamma^{-1} \circ \alpha_{2} \circ \gamma$.

С действием диэдральной группы на различных классах хордовых $n$ диаграмм (на классах соответствующих склеек) можно ознакомиться, например, в работах [3], [13], [2]. 


\section{3 Исчисление топологически неэквивалентных функций с одной вырожденной критической точкой типа седло на 2-сфере}

Как было отмечено ранее, для функций из класса $C_{M, m}\left(N_{g}\right)$ полным топологическим инвариантом является 2-цветная $O$-диаграмма с $n=2 g-1+M+m$ хордами, которая содержит $M$ черных (белых) и $m$ белых (черных) циклов.

Обозначим через $\Im_{M, m}^{n}$ класс 2-цветных диаграмм указанного типа. Тогда, как следует из работ [4] и [11], имеют место утверждения

Теорема А Число О-топологически неэквивалентных функиий из класса $C_{M, m}\left(N_{g}\right)$ равно числу неизоморфньх диаграмм из класса $\Im_{M, m}^{n}$.

Теорема В Число топологически неэквивалентных функиий из класса $C_{M, m}\left(N_{g}\right)$ равно числу неэквивалентных диаграмм из класса $\Im_{M, m}^{n}$.

Очевидно, что для функций из класса $C_{M, m}\left(S^{2}\right)$ (на двумерной сфере $\left.S^{2}\right)$ полным топологическим инвариантом является 2-цветная $O$-диаграмма с $n=M+m-1$ хордами, которая содержит точно $M$ черных (белых) и $m$ белых (черных) циклов. С учетом формулы (1), род такой диаграммы равен нулю. Поэтому функциям из класса $C_{M, m}\left(S^{2}\right)$ соответствую именно планарные 2-цветные $O$-диаграммы.

Таким образом, если через $C_{k, n-k+1}\left(S^{2}\right)$ обозначить класс гладких функций на 2-сфере, у которых кроме $k$ локальных максимумов и $n-k+1$ локальных минимумов только одна вырожденная критическая точка типа седло (индекс Пуанкаре которой равен $1-n$ ), то имеют место утверждения Предложение 1 Число О-топологически неэквивалентных функций из класса $C_{k, n-k+1}\left(S^{2}\right)$ равно числу неизоморфных диаграмм из класса $\Im_{k, n}$.

Предложение 2 Число топологически неэквивалентных функиий из класса $C_{k, n-k+1}\left(S^{2}\right)$ равно иислу неэквивалентных диаграмм из класса $\Im_{k, n}$. Пример 1 Среди неизоморфных диаграмм, приведенных на рис. 2: 1 из класса $\Im_{1,4}, 2$ из класса $\Im_{2,4}, 2$ из класса $\Im_{3,4}, 1$ из класса $\Im_{4,4}$.
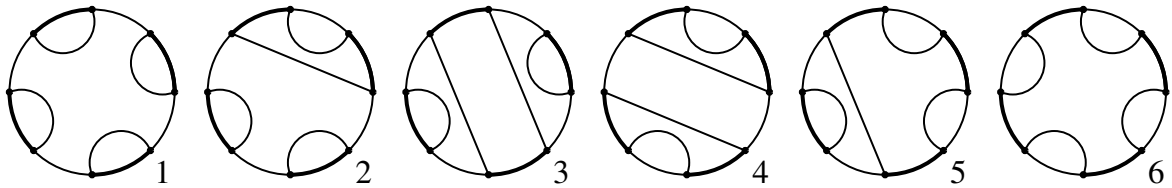

Рис. 2 все неизоморфные (неэквивалентные) 2-цветные планарные диаграммы $n=4$

Таким образом, существует 2 топологически неэквивалентные функиии из класса $C_{2,3}\left(S^{2}\right), 2$ - из класса $C_{3,2}\left(S^{2}\right), 1$ из $C_{1,4}\left(S^{2}\right)$ u 1 из $C_{4,1}\left(S^{2}\right)$. 


\section{4 Диаграммы из класса $\Im_{k, n}$ и «non-crossing partition»}

Определение 6 Разбиением $\pi_{k, n}$ множества $[n]=\{1,2, \ldots, n-1, n\}$ (partition of $[n]$ with $k$ blocks) называют совокупность $k$ непустых, попарно не пересекающихся подмножеств $\pi_{1}, \pi_{2}, \ldots \pi_{k}$ множества $[n]$, обгединение которых есть $[n]$. Подмножества $\pi_{i}$ принято называть блоками $\pi_{k, n}$.

Разбиение $\pi_{k, n}$ зачастую представляют в виде $\pi=\pi_{1} / \pi_{2} / \ldots / \pi_{k}$. Причем, принято считать, что внутри каждого блока элементы расположены в порядке возрастания, а сами блоки расположены в порядке возрастания их минимальных элементов (напр. [7]).

Определение 7 Разбиение называют «без самопересечений» (non-crossing partition) или же, коротко, планарным, если не существует элементов $a<b<c<d$ таких, что а и с содержатся в одном, a $b$ и $d$ в другом блоке. Множество всех таких разбиений обозначим как $N C P(n)$, а подмножество разбиений, содержащих точно $k$ блоков, - через $N C P_{k, n}$.

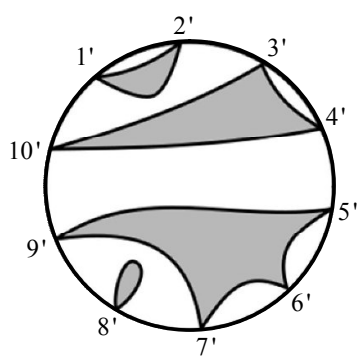

a)

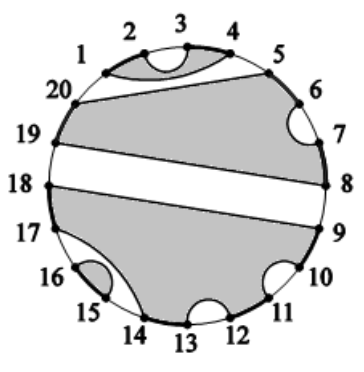

$\leftrightarrow$

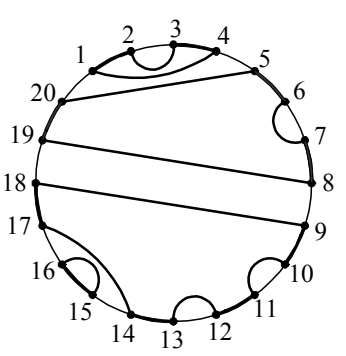

b)

Рис. 3 a) представление планарного разбиения $\pi=(1,2)(3,4,10)(5,6,7,9)(8)$ в виде круговой диаграммы с 10 вершинами; b) 2-цветная $O$-диаграмма рода 0 (построенная на 2-цветном $2 n$-шаблоне, $n=10$ ), соответствующая разбиению $\pi$

В виду биективности, которая очевидным образом возникает между разбиениями из множества $N C P_{k, n}$ и диаграммами из класса $\Im_{k, n}, 2$-цветную планарную диаграмму и соответствующее ей разбиение в дальнейшем будем отождествлять.

Более того, число неизоморфных (а также неэквивалентньх) диаграмм из класса $\Im_{k, n}$ равно числу неизоморфных (соответственно неэквивалентных) круговых диаграмм, отвечающих разбиениям из множества $N C P_{k, n}$. Причем в понятие изоморфности (эквивалентности) последних вкладывается такой же смысл как и для 2-цветных диаграмм (напр. [2]). 


\section{5 Перечисление диаграмм из класса $\Im_{k, n}$}

Введем следующие обозначения:

$P(n ; k)$ - число диаграмм из класса $\Im_{k, n}$, то есть $P(n ; k)=\left|\Im_{k, n}\right|=\left|N C P_{k, n}\right|$;

$P^{*}(n ; k)$ - число неизоморфных диаграмм из класса $\Im_{k, n}$;

$P^{* *}(n ; k)$ - число неэквивалентных диаграмм из класса $\Im_{k, n}$.

Известно, например из [8], что $\left|N C P_{k, n}\right|$ равна числу Нараяна $N(n ; k)$ («Narayana number»), которое определяется по формуле

$$
N(n ; k)=\frac{1}{n} C_{n}^{k} \cdot C_{n}^{k-1} .
$$

Так как $\forall n \in N$ справедливо равенство $N(n ; 1)=N(n ; n)=1$, то

$$
\begin{gathered}
P^{*}(n ; 1)=P^{*}(n ; n)=1, \\
P^{* *}(n ; 1)=P^{* *}(n ; n)=1 .
\end{gathered}
$$

Более того, так как

$$
N(n ; k)=\frac{1}{n} C_{n}^{k} \cdot C_{n}^{k-1}=\frac{1}{n} C_{n}^{n-k+1} \cdot C_{n}^{n-k}=N(n ; n-k+1),
$$

то для натуральных $n \geq k \geq 2$ имеют место и соотношения

$$
\begin{gathered}
P^{*}(n ; k)=P^{*}(n ; n-k+1), \\
P^{* *}(n ; k)=P^{* *}(n ; n-k+1) .
\end{gathered}
$$

5.1 Число неизоморфных диаграмм из класса $\Im_{k, n}$

Лемма 1 Для натуральных $n \geq k \geq 2$ число неизоморфных (неэквивалентных относительно действия ииклической группы порядка п) диаграмм из класса $\Im_{k, n}$ можсно вычислить с помощью соотношения

$$
\begin{aligned}
P^{*}(n ; k)= & \frac{1}{n}\left(\frac{1}{n} C_{n}^{k} \cdot C_{n}^{k-1}+\sum_{j \mid(n ; k), j \neq 1} \varphi(j)\left(\frac{n-k}{j}+1\right) \frac{1}{\frac{n}{j}} C_{\frac{n}{j}}^{\frac{k}{j}} C_{\frac{n}{j}}^{\frac{k}{j}-1}+\right. \\
& \left.+\sum_{j \mid(n ; k-1), j \neq 1} \varphi(j)\left(\frac{k-1}{j}+1\right) \frac{1}{\frac{n}{j}} C_{\frac{n}{j}}^{\frac{k-1}{j}+1} C_{\frac{n}{j}}^{\frac{k-1}{j}}\right),
\end{aligned}
$$

где $\varphi(q)$ - функиия Эйлера (количество натуральных чисел, не превосходящих q и взаимно простых с ним); $(s ; t)$ - наибольший общий делитель чисел s и $t$; а суммирование во втором и третвем слагаемых ведется по всем делителям (исключал 1-иу) чисел $(n ; k) u(n ; k-1)$ соответственно. 
Замечание 1 Учитьвая (5), соотношение (8) можно представить в виде

$$
\begin{aligned}
P^{*}(n ; k)= & \frac{1}{n}\left(N(n ; k)+\sum_{j \mid(n ; k), j \neq 1} \varphi(j)\left(\frac{n-k}{j}+1\right) \cdot N\left(\frac{n}{j} ; \frac{n-k}{j}+1\right)+\right. \\
& \left.+\sum_{j \mid(n ; k-1), j \neq 1} \varphi(j)\left(\frac{k-1}{j}+1\right) \cdot N\left(\frac{n}{j} ; \frac{k-1}{j}+1\right)\right) .
\end{aligned}
$$

Доказательство Все диаграммы из класса $\Im_{k, n}$, которые инвариантны относительно поворота на некоторый угол $\omega_{j}=\frac{2 \pi}{j}, j \neq 1$ («самосовмещаются» при повороте около центра шаблона на угол $\omega_{j}$ в направлении движения часовой стрелки) разобьем на диаграммы двух типов.

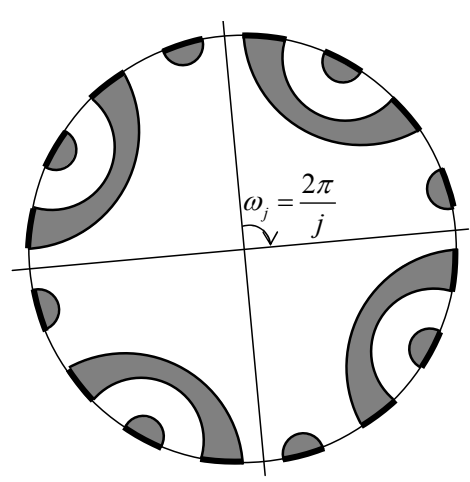

a)

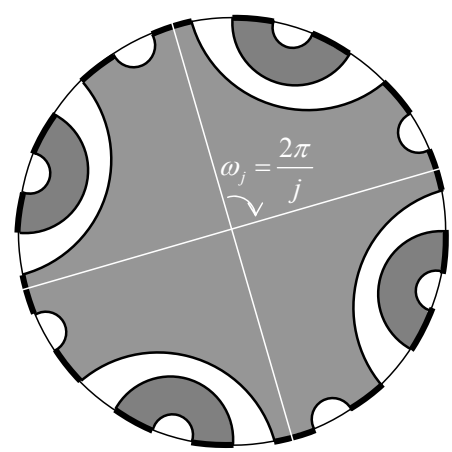

b)

Рис. 4 a) планарная 16-диаграмма I типа; b) планарная 16-диаграмма II типа

I типа - те, которые не содержат черного цикла («черного полигона»), инвариантного относительно поворота на некоторый угол. Очевидно, что в этом случае число $j$ должно быть общим делителем чисел $n$ и $k$ - рис. $1 a)$.

II типа - те, которые содержат черный цикл («черный полигон»), инвариантный относительно поворота на некоторый угол. Очевидно, что в этом случае число $j$ должно быть общим делителем чисел $n$ и $k-1$ - рис. $1 b)$.

Тогда, применяя лемму Бернсайда, величину $P^{*}(n ; k)$ можно представить в виде

$$
\begin{gathered}
P^{*}(n ; k)= \\
=\frac{1}{n}\left(N(n ; k)+\sum_{\substack{j \mid(n ; k), j \neq 1}} \varphi(j) \rho_{1}(n ; k ; j)+\sum_{\substack{j \mid(n ; k-1), j \neq 1}} \varphi(j) \rho_{2}(n ; k ; j)\right),
\end{gathered}
$$


где $\rho_{1}(n ; k ; j), \rho_{2}(n ; k ; j)$ - число диаграмм I и II типа соответственно, которые инвариантны относительно поворота на угол $\omega_{j}(j \neq 1)$.

Так как $N(n ; k)=N(n ; n-k+1), P^{*}(n ; k)=P^{*}(n ; n-k+1)$, то с учетом того факта, что для произвольных натуральных $a>b$ справедливо равенство $(a ; b)=(a ; a-b)$, имеем

$n \cdot P^{*}(n ; k)=N(n ; k)+$

$$
+\left(\sum_{j \mid(n ; k), j \neq 1} \phi(j) \rho_{1}(n ; k ; j)+\sum_{j \mid(n ; k-1), j \neq 1} \phi(j) \rho_{2}(n ; k ; j)\right)=
$$

$=n \cdot P^{*}(n ; n-k+1)=N(n ; n-k+1)+$

$+\left(\sum_{\substack{j \mid(n ; n-k+1), j \neq 1}} \varphi(j) \rho_{1}(n ; n-k+1 ; j)+\sum_{\substack{j \mid(n ; n-k), j \neq 1}} \varphi(j) \rho_{2}(n ; n-k+1 ; j)\right)=$

$=N(n ; k)+\left(\sum_{\substack{j \mid(n ; k-1), j \neq 1}} \varphi(j) \rho_{1}(n ; n-k+1 ; j)+\sum_{\substack{j \mid(n ; k), j \neq 1}} \varphi(j) \rho_{2}(n ; n-k+1 ; j)\right)$

Из соотношений (11) и (12) получаем равенство

$$
\begin{gathered}
\sum_{\substack{j \mid(n ; k), j \neq 1}} \varphi(j) \rho_{1}(n ; k ; j)+\sum_{\substack{j \mid(n ; k-1), j \neq 1}} \varphi(j) \rho_{2}(n ; k ; j)= \\
=\sum_{\substack{j \mid(n ; k), j \neq 1}} \varphi(j) \rho_{2}(n ; n-k+1 ; j)+\sum_{\substack{j \mid(n ; k-1), j \neq 1}} \varphi(j) \rho_{1}(n ; n-k+1 ; j),
\end{gathered}
$$

которое позволяет выдвинуть гипотезу о том, что при (допустимых) фиксированных $n, k, j$ величины $\rho_{1}(n ; k ; j)$ и $\rho_{2}(n ; n-k+1 ; j)$, а также $\rho_{2}(n ; k ; j)$ и $\rho_{1}(n ; n-k+1 ; j)$ совпадают.

Убедимся в справедливости выдвинутой гипотезы.

$\mathrm{C}$ учетом введенной терминологии и обозначений, величина $\rho_{1}(n ; k ; j)$ определяет число всех тех диаграмм (из класса $\Im_{k, n}$ ), которые инвариантны относительно поворота на угол $\omega_{j}=\frac{2 \pi}{j}$ и не содержат черного цикла («черного полигона»), инвариантного относительно поворота на угол $\omega_{j}$. Но тогда каждая такая диаграмма (с $k$ черными и $n-k+1$ белыми циклами) содержит белый цикл («белый полигон»), инвариантный относительно поворота на угол $\omega_{j}$.

Если черные дуги перекрасить в белый цвет, а белые дуги - в черный цвет, то каждая из упомянутых выше диаграмм будет 
- инвариантной относительно поворота на такой же угол $\omega_{j}$;

- содержать $n-k+1$ черных циклов, среди которых один черный цикл («черный полигон»), инвариантный относительно поворота на угол $\omega_{j}$.

Тогда очевидно, что при фиксированных $n, k, j$ выполняется равенство

$$
\rho_{1}(n ; k ; j)=\rho_{2}(n ; n-k+1 ; j) \text {, }
$$

а, с учетом (13), - и равенство

$$
\rho_{2}(n ; k ; j)=\rho_{1}(n ; n-k+1 ; j) .
$$

Таким образом, если $\rho_{1}(n ; k ; j)$ обозначить как $\rho(n ; k ; j)$, то, с учетом (14) и (15), имеет место равенство

$$
\rho_{2}(n ; k ; j)=\rho(n ; n-k+1 ; j) .
$$

И поэтому доказательство леммы сводится к доказательству того факта, что число $\rho_{1}(n ; k ; j)$ (всех) диаграмм I типа, инвариантных относительно поворота на угол $\omega_{j}$ (неподвижных относительно действия соответствующего элемента циклической группы), можно вычислить по формуле

$$
\rho_{1}(n ; k ; j)=\left(\frac{n-k}{j}+1\right) \frac{1}{\frac{n}{j}} C_{\frac{n}{j}}^{\frac{k}{j}} C_{\frac{n}{j}}^{\frac{k}{j}-1} .
$$

В работе [10] (Theorem 7.2) установлено, что при $j \geq 2$ число $\left[N\left(n ; k^{\prime}\right)\right]$ разбиений из класса $N C P_{k, n}$, которые имеют ранг $k^{\prime}$ (в наших терминах $k=n-k^{\prime}$ блоков) и являются инвариантными относительно поворота на угол $\omega_{j}=\frac{2 \pi}{j}$, можно вычислить по формуле

$$
\left[N\left(n ; k^{\prime}\right)\right]=\frac{n-k^{\prime}}{n} \cdot\left(C_{\frac{n}{j}}^{\frac{k^{\prime}}{j}}\right)^{2} .
$$

Поэтому

$$
\begin{aligned}
& \rho_{1}(n ; k ; j)=[N(n ; n-k)]=\frac{n-(n-k)}{n} \cdot\left(C_{\frac{n}{j}}^{\frac{n-k}{j}}\right)^{2}=\frac{k}{n} \cdot C_{\frac{n}{j}}^{\frac{n-k}{j}} \cdot C_{\frac{n}{j}}^{\frac{n-k}{j}}= \\
& =\frac{k}{n} \cdot C_{\frac{n}{j}}^{\frac{k}{j}} \cdot C_{\frac{n}{j}}^{\frac{k}{j}}=\frac{k}{n} \cdot C_{\frac{n}{j}}^{\frac{k}{j}} \cdot \frac{\frac{n-k}{j}+1}{\frac{k}{j}} \cdot C_{\frac{n}{j}}^{\frac{k}{j}-1}=\left(\frac{n-k}{j}+1\right) \cdot \frac{1}{\frac{n}{j}} C_{\frac{n}{j}}^{\frac{k}{j}} C_{\frac{n}{j}}^{\frac{k}{j}-1} .
\end{aligned}
$$

Следствие 1 Для произвольного простого $n$ число неизоморфных диаграмм из класса $\Im_{k, n}$ можно вычислить посредством соотношений

$$
P^{*}(n, k)= \begin{cases}\frac{1}{n^{2}} C_{n}^{k} C_{n}^{k-1}+\frac{n-1}{n}, & k=1 ; n \\ \frac{1}{n^{2}} C_{n}^{k} C_{n}^{k-1}, & k \neq 1 ; n\end{cases}
$$


Замечание 2 Следует отметить, что начальные значения величины $P^{*}(n, k)$ для натуральных $1 \leq k \leq n \leq 9$ совпадают с членами последовательности A209805 ( Triangle read by rows: $T(n, k)$ is the number of $k$-block noncrossing partitions of $n$-set up to rotations») [10], полученных автором (Tilman Piesk, March 10 2012) программным путем.

Кроме того, с учетом резулътатов работы [2], имеет место равенство

$$
\sum_{k=1}^{n} P^{*}(n, k)=p_{n}^{*}=\frac{1}{n}\left(\frac{1}{n+1} C_{2 n}^{n}+\sum_{\substack{1 \leq i<n, i \mid n}} \varphi\left(\frac{n}{i}\right) C_{2 i}^{i}\right)
$$

где $p_{n}^{*}$ - число неизоморфных разбиений из класса $N C P(n)$, или, что тоже, - число неизоморфных 2-цветных планарных -диаграмм.

5.2 Число неэквивалентных диаграмм из класса $\Im_{k, n}$

Применяя лемму Бернсайда, число неэквивалентных диаграмм из класса $\Im_{k, n}$ можно вычислить с помощью соотношения

$$
P^{* *}(n, k)=\frac{1}{2}\left(P^{*}(n, k)+\frac{1}{n} \cdot P_{n, k}^{s i m}\right),
$$

где $P_{n, k}^{\text {sim }}$ - число тех диаграмм из класса $\Im_{k, n}$, которые являются симметричными относительно хотя бы одной из $n$ различных осей симметрии 2-цветного $2 n$-шаблона. Причем

$$
P_{n, k}^{s i m}= \begin{cases}n \cdot p_{0}(n, k), & n=2 m \pm 1 \\ \frac{n}{2} \cdot\left(p_{1}(n, k)+p_{2}(n, k)\right), & n=2 m,\end{cases}
$$

где $p_{0}(n, k)$ - число диаграмм из $\Im_{k, n}$, симметричных относительно фиксированной оси симметрии, проходящей через середины диаметральнопротивоположных черной и белой дуг 2 -цветного $2 n$-шаблона;

$p_{1}(n, k)\left(p_{2}(n, k)\right)$ - число диаграмм из $\Im_{k, n}$, симметричных относительно фиксированной оси симметрии, проходящей через середины диаметральнопротивоположных черных (соответственно белых) дуг $2 n$-шаблона.

Хорошо известно (напр. из [8]), что существует биективное соответствие между элементами множества $N C P_{k, n}$ (а потому и $\Im_{k, n}$ ) и элементами множества, известными как «Dyck $n$-paths with exactly $k$ peaks». Как следует из работ [1] и [2], величина $T(n, k)=\frac{1}{n} \cdot P_{n, k}^{s i m}$ совпадает с числом объектов, именуемых как «symmetric Dyck paths of semi-length $n$ with $k$ peaks» 
(последовательность $A 088855$ в [10]). Более того, в работе [1] (Example 37 ) показано, что

$$
T(n, k)=C_{\left[\frac{n-1}{2}\right]}^{\left[\frac{k-1}{2}\right]} \cdot C_{\left\lceil\frac{n-1}{2}\right\rceil}^{\left\lceil\frac{k-1}{2}\right\rceil},
$$

где $[q]$ - целая часть числа $q$ (определяемая как наибольшее целое, меньшее или равное $q) ;\lceil q\rceil$ - функция «потолок», определяемая как наименьшее целое, большее или равное $q$.

С учетом соотношений (21) и (22), имеет место

Лемма 2 Для натуральных $n \geq k \geq 2$ число неэквивалентных (относительно действия диэдральной группы порядка 2n) диаграмм из класса $\Im_{k, n}$ можно вычислить с помощью соотношения

$$
P^{* *}(n, k)=\frac{1}{2}\left(P^{*}(n, k)+C_{\left\lfloor\frac{n-1}{2}\right\rfloor}^{\left\lfloor\frac{k-1}{2}\right\rfloor} \cdot C_{\left\lceil\frac{n-1}{2}\right\rceil}^{\left\lceil\frac{k-1}{2}\right\rceil}\right),
$$

где $\lfloor q\rfloor=\max \{n \in Z \mid n \leq q\},\lceil q\rceil=\min \{n \in Z \mid n \geq q\}$, a $P^{*}(n, k)$ определяетсл по бормуле (8).

Заметим, что начальные значения величины $P^{* *}(n, k)$ совпадают с членами последовательности $A 209612$ [10].

\section{Результаты и выводы}

На основании Предложений 1 и 2, Леммы 1 и Леммы 2 имеют место основные результаты работы

Теорема 1 Для натуральных $n \geq k \geq 2$ число О-топологически неэквивалентных функиий из класса $C_{k, n-k+1}\left(S^{2}\right)$ можно вычислить с помощью бормульь (8).

Теорема 2 Для натуральных $n \geq k \geq 2$ число топологически неэквивалентных функций из класса $C_{k, n-k+1}\left(S^{2}\right)$ можно вычислить с помощью формульь (23).

Начальные значения числа О-топологически и топологически неэквивалентных функций из класса $C_{k, n-k+1}\left(S^{2}\right)$ для натуральных $2 \leq k \leq n \leq 18$ приведены в таблицах 1 и 2 соответственно.

Кроме того, в представленной статье впервые приводится решение задачи (более известной под названием) «Enumeration of non-crossing partitions of $[n]$ with $k$ blocks under rotation and reflection», результаты которой были анонсированы в материалах «XV международной научной конференции им. академика Михаила Кравчука» и международной конференции «Геометрия в Одессе - 2014». 


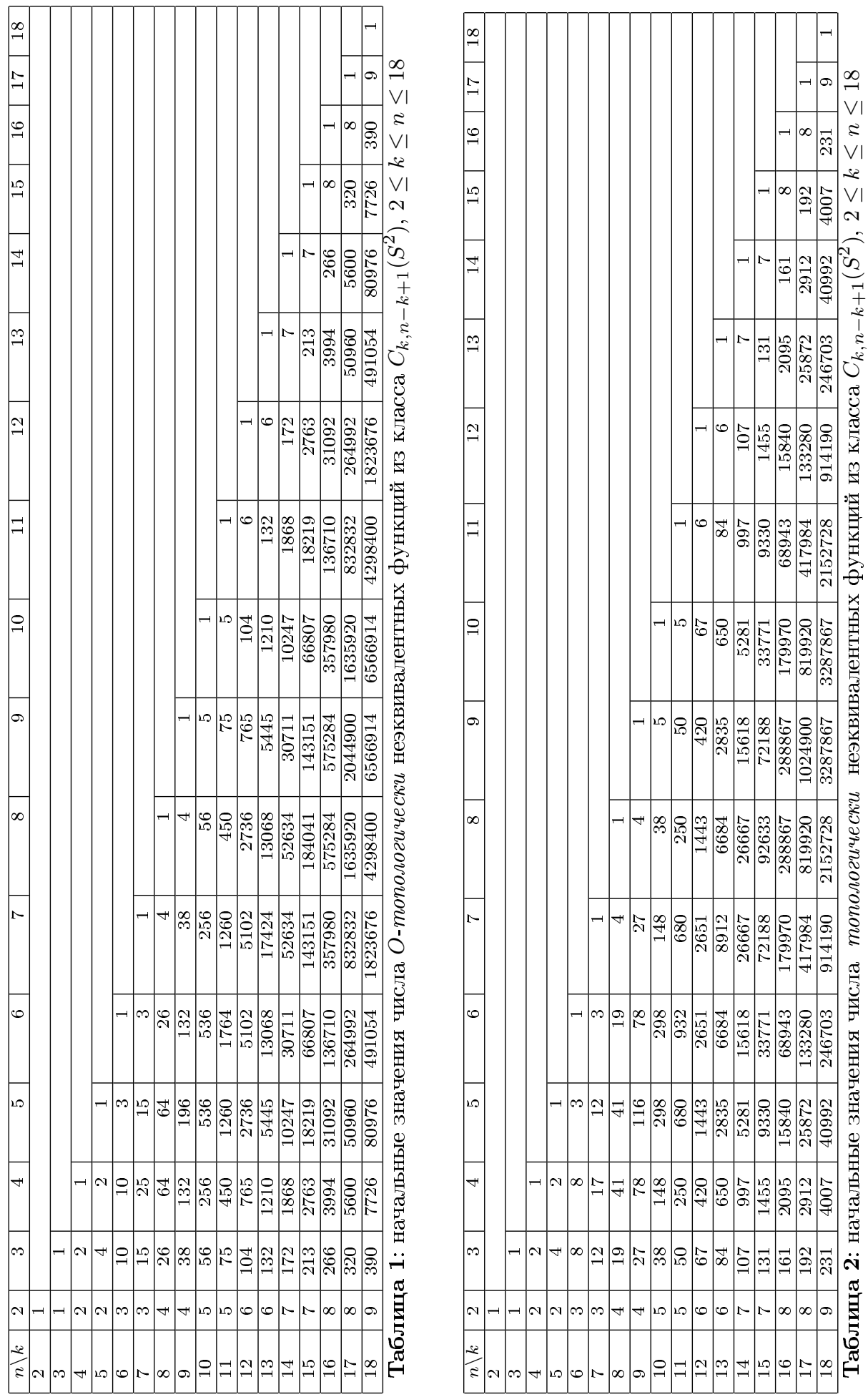




\section{Список литературы}

1. Barry P. On Integer-Sequence-Based Constructions of Generalized Pascal Triangles // Journal of Integer Sequences. - 2006. - V. 9, № 2. - Article 06.2.4, 34 pp. (electronic)

2. Callan D. Non-crossing Partitions under Rotation and Reflection / D. Callan, L. Smiley // http://arxiv.org/abs/math.CO/0510447, 2005.

3. Cori R. Counting non-isomorphic chord diagrams / R. Cori, M. Marcus // Theoretical Computer Science. - 1998. - V. 204 Issue 1-2. - P. 55-73.

4. Kadubovsky A. Classification of O-topolodically non-equivalent function with color chord diagrams / A. Kadubovsky, A. Klimchuk // Method of functional analysis and topology. - 2004. - Vol. 10, №3. - P. 23-32.

5. Prishlyak A. O. Topological equivalence of smooth functions with isolated critical points on a cloused surface / A.O. Prishlyak / Topology and its Aplications. - 2002. - Vol. 119, № 3. - P. 257-267.

6. Reiner V. The Cyclic Sieving Phenomenon / V. Reiner, D. Stanton, D. White // Journal of Combinatorial Theory, Series A 108. - 2004. - P. 17-50.

7. Simion R. Noncrossing partitions / R. Simion // Discrete Mathematics. - 2000. Vol. 217. - P. 367-409.

8. Stanley R. Enumerative combinatorics // Volume 2, Cambridge Univ. Press, New York, 1999.

9. Stanley R. Catalan Addendum// 6 June, 2010.

10. The OEIS Foundation Inc., «The On-Line Encyclopedia of Integer Sequences», published electronically at http://oeis.org.

11. Кадубовський О. Топологічна еквівалентність функиій на орієнтованих поверхняx / О. Кадубовський // Український математичний журнал. - 2006. - Т. 58, № 3. - С. 343-351.

12. Кадубовський О. Про один клас хордових діаграм максималъного роду / О. Кадубовський // Вісник Київського університету ім. Тараса Шевченка. Серія: Фізикоматематичні науки. - 2006. - Вип. 1. - С. 17-27.

13. Кадубовський О. А. Двокольорові $O-i N$-діаграми / О.А. Кадубовський, О.В. Сторожилова, Н.В. Сторожилова // Пошуки і знахідки. Серія: фізико-математичні науки. - 2010. - Вип. 10, Том I. - С. 41-50.

14. Кадубовський О. А. Про число топологічно нееквівалентних функцій з однією виродженою критичною точкою типу сідла на двовимірній сфері / О.А. Кадубовський // Геометрія та топологія функцій: Збірник праць Інституту математики НАН України. - Т 7, № 4, 2010. - С. 87-107.

15. Кадубовський О. А. Про один клас гладжих функцій на двовимірній сфері / О.А. Кадубовський // Вісник СДПУ. Математика. - Слов'янськ: СДПУ. - Вип. 1(4), 2010. - С. 39-57.

16. Кадубовський О. А. Двокольорові хордові О-діаграми мінімального роду / О.А. Кадубовський, Ю.В. Гладищук // Збірник наукових праць фізико-математичного факультету СДПУ. - 2011. - Вип. 1. - С. 49-60.

17. Кадубовський О. А. Двокольорові хордові О-діаграми мінімалъного роду з $k=9$ ииклами певного колъору / О.А. Кадубовський, К.В. Хабарова, Ю.В. Сапсай // Збірник наукових праць фізико-математичного факультету ДДПУ. - 2014. - Вип. 4. - C. 69-84.

18. Шарко В. В. Гладкая и топологическал эквивалентность функций на поверхностлх / В.В. Шарко // Український математичний журнал. - 2003. - Т. 55, № 5. C. $687-700$.

\section{Александр Анатольевич Кадубовский}

ДГПУ, Славянск, Украина

E-mail: kadubovs@ukr.net 


\begin{abstract}
Aleksandr An. Kadubovskyi
Donbass State Teachers' Training University, Slavyansk, Ukraine.
\end{abstract}

On the number of topologically non-equivalent functions with one degenerate saddle critical point on two-dimensional sphere, II

In this paper we consider the smooth functions with three critical values on twodimensional sphere $S^{2}$, that possess only one (degenerate) saddle critical point in addition to $M$ local maxima and $m$ local minima. For any natural $M$ and $m$ we calculate the number of topologically non-equivalent such functions. 